\title{
Review of the Book "Magnetooptical Spectroscopy of the Rare-Earth Compounds: Development and Application”
}

\section{S. Edelman}

L.V. Kirensky Institute of Physics, Siberian Branch of Russian Academy of Sciences, Krasnoyarsk, Russia. Email: ise@iph.krasn.ru

Received March 16 ${ }^{\text {th }}, 2012$; revised April 12 $2^{\text {th }}, 2012$; accepted April 20 ${ }^{\text {th }}, 2012$

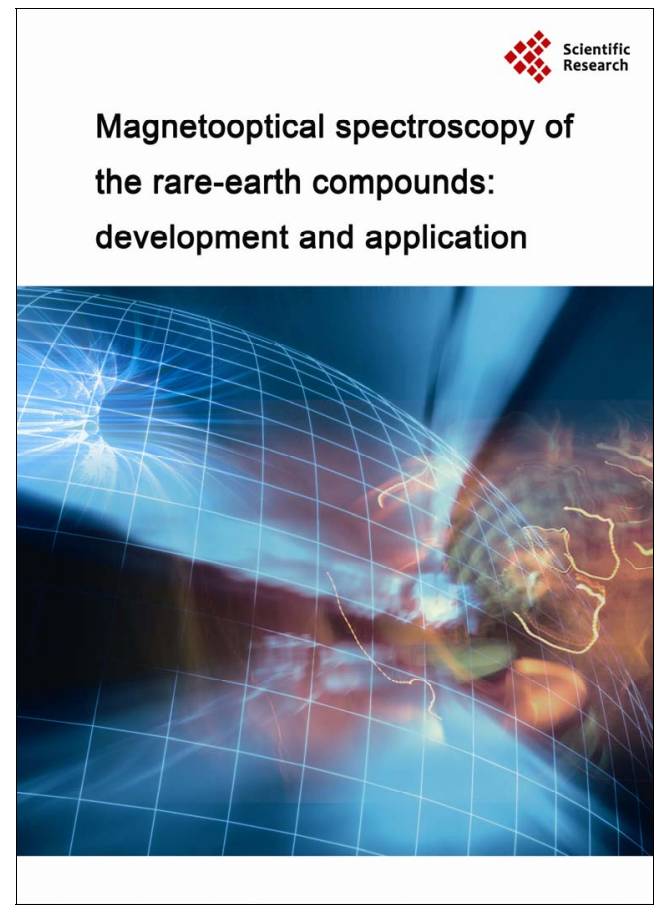

ISBN: 978-1-935068-59-4

139pp Pub.date: January 2012

Price: US \$89

This book reviews and provides numerous theoretical calculations for the first time relevant to the optics and magneto-optics of the trivalent rare earth ions $\left(\mathrm{RE}^{3+}\right)$ and their compounds. The $\mathrm{RE}^{3+}$ have unique spectroscopic properties that make them exceptionally attractive to both scientists, who probe the fundamental properties of these systems, and engineers, who develop these systems into photonic devices eagerly marketed in expanded technologies. Magneto-optical effects in compounds doped with $\mathrm{RE}^{3+}$ have been studied since the dawn of modern physics and have had a profound impact on their development in terms of practical opto-electronic devices. The authors of this new, exciting, and fresh text are world famous in this field, with over 150 years of experience in developing important theoretical and experimental con- tributions and over fifty years of more than 500 refereed publications in this field in the Soviet Union and the United States. They offer both a conceptual description of magneto-optical phenomena, as well as clear, detailed theoretical development published for the first time of many of the phenomena only briefly addressed in the literature. The book is based on the author's original work in theoretical and experimental investigations in magneto-optics. Numerous graphs and simple examples model the phenomena described in the text, making it a highly desirable treatise for scientists beginning their studies in the field of magneto-optics.

\section{The Book Consists of Four Chapters}

Chapter one describes the key physical properties of the electronic structure and the energy-level spectrum of the $\mathrm{RE}^{3+}$ both in the free-ion state and in two representative types of crystals (garnets and ortho-aluminates). These two crystalline hosts are currently the most sought after systems for modern practical applications. Discussion is developed in detail on the crystal electric field that removes the degeneracy of the $\mathrm{RE}^{3+}$ energy levels in the lattice and the magnetic features of these two $\mathrm{RE}^{3+}$-doped paramagnetic compounds having either Kramers or non-Kramers energy sublevels (Stark levels) depending on whether $\mathrm{RE}^{3+}$ is an odd-number or even-number electron system in the $4 \boldsymbol{f}^{n}$ sub-shell. Systematics of $\mathrm{RE}^{3+}$ garnets and ortho-aluminates are presented, with special attention paid to the description of the strongly anisotropic ("Ising") character found in the magnetic behavior of some $\mathrm{RE}^{3+}$.

Chapter 2 is devoted to the optics of the $4 \mathrm{f} \rightarrow 4 \mathrm{f}$ and $4 \mathrm{f} \rightarrow 5 \mathrm{~d}$ transitions in crystals. Current methods are used to calculate $\mathrm{RE}^{3+}$ transition energies and intensities of the forbidden $4 \mathrm{f} \rightarrow$ 4f transitions in RE compounds. Results obtained from recently developed experimental methods used to measure the optical features of RE compounds (such as hot-band spectroscopy and site-selective polarized spectroscopy) are interpreted in detail by applying group theory to the analyses of the optical spectra of 
several RE ions in crystal field environments of low symmetry. Also, a theoretical description of the optical spectra associated with the allowed $4 \mathrm{f} \rightarrow 5 \mathrm{~d}$ transitions in the $\mathrm{Tb}^{3+}$ and $\mathrm{Ce}^{3+}$ garnets is analyzed within the framework of a "strong-weak" crystalline field approximation.

Chapter 3 is devoted to the phenomenological theory of magnetooptical phenomena that is general for all rare-earth dielectrics. A quantum-mechanical theory for the linear magnetooptical effects in paramagnetic RE garnets due to the allowed electric-dipole $4 \mathrm{f} \rightarrow 5 \mathrm{~d}$ transitions is considered in detail in the approximation of an "average" crystalline field. The theory of the Faraday effect is also applied to magnetic-dipole transitions in several $\mathrm{RE}^{3+}$. The role of the Van-Vleck contribution to the mixing of states in the circular magneto-optics of the $\mathrm{Eu}^{3+}, \mathrm{Sm}^{3+}$ and $\mathrm{Gd}^{3+}$ in the garnets is discussed in detail. The special features of the Faraday effect (FE) and magnetic circular dichroism (MCD) in YAG activated by the $\mathrm{Tb}^{3+}$ and $\mathrm{Ce}^{3+}$ are considered in the approximation of a "strong-weak" crystalline field within the ultraviolet spectral range. The nature of the magneto-optical activity (MOA) mechanism in the RE compounds with orthoaluminate structure is discussed using group theory as the tool for analysis. As a separate topic, the appearance of the so-called "Anti-Zeeman" effect is considered in the absorption spectra of terbium-gallium garnet TbGG in a

\section{To order: http://www.scirp.org/book/ bookorder@scirp.org}

magnetic field.

The final chapter covers the physics of the magnetooptical effects in radiative $4 \mathrm{f} \rightarrow 4 \mathrm{f}$ transitions in $\mathrm{RE}$ compounds. Starting with the basic ideas concerning these effects, the authors present a set of experimental results for the degree of magnetic circular polarization luminescence (MCPL) of $\mathrm{RE}^{3+}$ in garnets, and they also analyze the features in the MCPL spectra in depth. The authors are the recognized authorities in this relatively new field of magneto-optics.

In the conclusive part authors give the reader an idea of the recent (2010 and 2011) technological activity derived from the results and predictions reported in the book and in other author publications.

The text of the book is well organized and clearly presented. In my opinion it will be of great use both for those who study the optical and magneto-optical properties of the RE compound professionally and for those who intend to obtain general ideas on these phenomena.

\section{REFERENCES}

[1] U. V. Valiev, J. B. Gruber and G. W. Burdick, "Magnetooptical Spectroscopy of the Rare-Earth Compounds: Development and Application," Scientific Research Publishing, USA, 2012. 